Extended article since the oral presentation at the ERCOFTAC international symposium «Unsteady separation in fluid-structure interaction» Mykonos, Greece, June 17-21, 2013

\title{
VMS AND OES-BASED HYBRID SIMULATIONS OF BLUFF BODY FLOWS
}

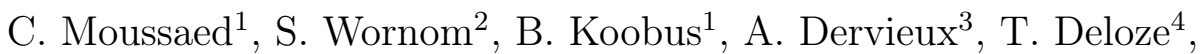 \\ R. El Akoury ${ }^{5}$, D. Szubert ${ }^{6}$, Y. Hoarau ${ }^{7}$, M. Braza ${ }^{6}$ \\ ${ }^{1}$ I3M, université de Montpellier 2, CC051, 34095 Montpellier, France \\ ${ }^{2}$ Lemma, 2000 route des Lucioles, 06410 Biot, France \\ ${ }^{3}$ INRIA, 2004 route des Lucioles, 06902 Sophia-Antipolis, France \\ ${ }^{4}$ AREVA, France \\ ${ }^{5}$ Scientific Research Center in Engineering (CRSI), \\ Faculty of Engineering, Lebanese University, Lebanon, \\ former:Institut de Mécanique des Fluides de Toulouse \\ ${ }^{6}$ Institut de Mécanique des Fluides de Toulouse, Unité Mixte C.N.R.S.-I.N.P.T. 5502, \\ avenue du Professeur Camille Soula, 31400 Toulouse, France \\ ${ }^{7}$ ICUBE-Strasbourg, 2 rue Boussingault, 67000 Strasbourg, France
}

\begin{abstract}
.
The present article focuses on the improvement of the two components of hybrid RANS/LES models. In the LES component, a Variational Multiscale (VMS) formulation [17] is introduced with the dynamic control of Germano et al, [11]. In the RANS component, an Organised Eddy Simulation (OES) model is used, [4,39]. The impact of these modifications on several critical flows past bluff bodies is analysed. These are the flow past cylinder at $R e=140,000$ entering the critical regime because of blockage ratio confinement, a flow past a cylinder at $R e=1,000,000$, as well as the flow past a tandem cylinder at $R e=166,000$.
\end{abstract}

Key words: hybrid turbulence models, RANS, VMS-LES, OES, DDES, bluff body flows.

\section{Introduction}

In the context of high Reynolds number flows around bodies, the pioneering studies of Roshko (1961) [35], Wieselberger (1921) [47], Bearman (1965) [3] and Schewe (1983) [37] had enlightened the transition into the critical regime, associated with the drastic decrease of drag in the range of Reynolds numbers $2.10^{5}$ and $5.10^{5}$ around a circular cylinder, followed by a drag increase afterwards, in the supercritical regime, in which the vortex shedding disappears in a specific Reynolds number range, to reappear afterwards. These changes are linked to the transition in the boundary layer upstream of the separation, which progressively moves in the separated shear layer downstream with a simultaneous movement of the separation points downstream . Then, the further increase of drag in the supercritical regime is associated with the separation point motion in more upstream positions. More precisely, in the passage to the critical regime, there are two discontinuous abrupt drag decreases 
and the existence of asymmetric forces on the cylinder surface (Bearman, 1965; Schewe, 1983). The passage to the critical and supercritical regime highly interests the aeronautics design and from a fundamental research point of view, the reasons of vortex shedding disappearance in a specific supercritical Reynolds number range. The numerical simulation of the drag crisis and of the supercritical regime remain important CFD challenges. The supercritical regime offers the possibility of the boundary layer treatment as fully turbulent, which is a simpler situation in respect for turbulence modelling issues than the transitional regime corresponding to the drag crisis. In this context, LES approaches and advanced URANS and hybrid RANS-LES methods can be adapted in order to capture the boundary layer nature and the transition location. LES methods need a quite high number of degrees of freedom (of order 100-500 Million for the single cylinder's problem, Rolfo-Revell et al, (2013) [34], Rodriguez et al, 2013 [18]), whereas hybrid methods are generally more economic as seen in the collected articles of the 4th Hybrid RANS-LES methods symposium (Girimaji et al, 2015 [1]). The objective of the present article is to offer reliable hybrid methods needing relatively economic grids.

\section{Methodology}

The widely used RANS models usually have difficulties in providing accurate predictions for flows with massive separation, as for instance the flow around bluff bodies. An alternative approach is the Large-Eddy Simulation (LES), which, for massively separated flows, is generally more accurate, but also computationally more expensive, than RANS. Indeed the grid needs to be sufficiently fine to resolve a significant part of the turbulent scales, and this becomes particularly critical in the near-wall regions. Moreover, the cost of LES increases with increasing Reynolds number. In this context, hybrid strategies have been proposed in the literature, which combine RANS and LES approaches together (see [36, 9] for a review). However, two main issues have to be addressed for the improvement of hybrid strategies as industrial tools. First they have to produce usable predictions with rather coarse meshes, second, they have to yield more accurate predictions.

The present study concentrates on the improvement of the two components of hybrid RANS/LES models. Concerning the RANS component, an Organised Eddy Simulation (OES) model is used, [5, 4, 39]. The impact of these modifications on the accurate prediction of three critical flows past bluff bodies is analysed. Concerning the LES component, a Variational Multiscale (VMS) formulation [17] is introduced and applied to high-Reynolds bluff body flows.

Hybrid with OES: the unsteady turbulent flow around bodies at high Reynolds number can be predicted by an anisotropic eddy-viscosity model in the context of the Organised Eddy Simulation (OES). A tensorial eddy-viscosity concept similar to ARSM [10] has been developed in [4], to reinforce turbulent stress anisotropy, that is a crucial characteristic of non-equilibrium turbulence in the near-region. The theoretical aspects of the modelling were investigated by means of a phaseaveraged Time-Resolved PIV in the flow around a circular cylinder at Reynolds number 140,000, the so called "IMFT circular cylinder" test case in the context of the European research program DESIDER (Detached Eddy Simulation for Industrial Aerodynamics, Haase et al, 2009). This test-case of a circular cylinder in a confined 

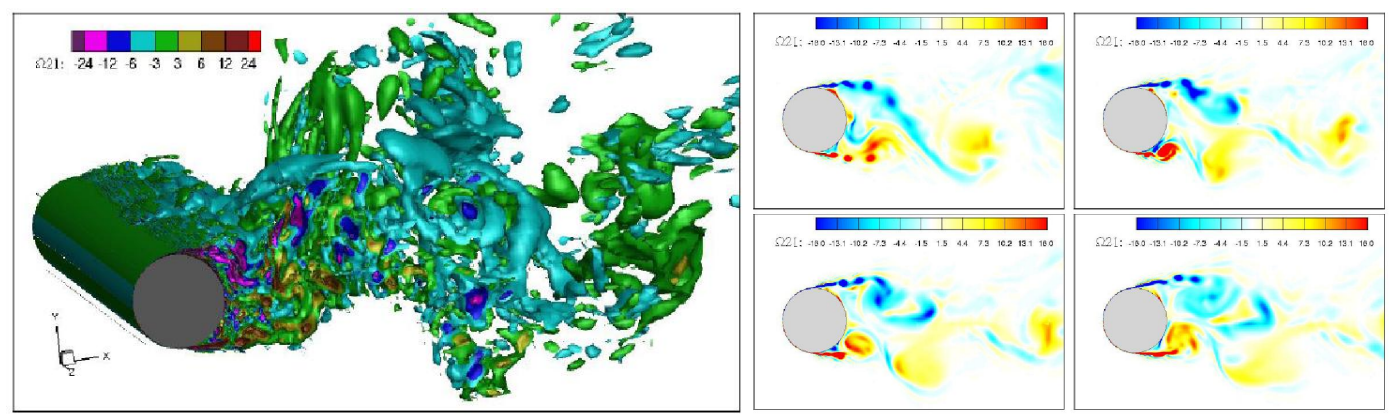

Figure 1: Flow past a cylinder, $R e=140,000$, the "IMFT circular cylinder" test case, DESIDER-EU program. Left: $\Omega_{z}$ vorticity iso-contours and aspect ratio 4.85 by means of the DDES-k-omega-OES modelling. Right: Tracking of the Kelvin-Helmholtz eddies and of their interaction (merging) into the von Kármán vortices.

environment was chosen in order to allow simulations with reasonable grid sizes, using the exact experimental boundary conditions and especially avoiding "infinite" - spanwise conditions as in former experiments by Cantwell and Coles (1983) [6]. The blockage ratio in the DESIDER test-case is of 0.208 and the aspect ratio of 4.85. The Time-resolved two-and three-component PIV measurements were carried out in the S4 subsonic wind tunnel of IMFT, Perrin (2005), Perrin et al $(2007,2008)$ and used by a number of academic and industrial partners in the DESIDER program, as well as by further studies after this program [30, 32, 31]. The present blockage ratio makes the flow entering the critical regime at Reynolds number 140,000 contrarily to previous experiments in the literature. A pronounced stress-strain misalignment was quantified in the near-wake region of the detached flow, that is well captured by a tensorial eddy-viscosity concept. This is achieved by modelling the turbulence stress anisotropy tensor by its projection onto the principal directions of the strain-rate tensor. Additional transport equations for the projection coefficients are derived from a second-order moment closure scheme. The modification of the turbulence length scale yielded by OES is introduced as the RANS length scale in the Detached Eddy Simulation hybrid modelling approaches (DES and DDES), originally proposed by Spalart et al, (1997) and (Spalart et al, 2006) respectively, $[42,41,40]$. This blending leads to the so-called DDES-OES modelling, successfully used in complex bluff body flows involving also multiple obstacles and vibrational instabilities (Shinde et al, 2014). This blending allows a smooth passage from the near-wall region handled by OES towards the LES region handled by the DDES and avoids the well known problem of MST, "Modeled Stress Depletion", which occurs whenever the LES region approaches too much the near-wall area. Even in case of standard DDES use, this "grey area" may develop around the body as discussed by Grossi et al, (2014), [13].

Hybrid turbulence modelling with VMS-Dynamic: The RANS closure terms provided by a RANS and a SGS eddy-viscosity model are blended together through the introduction of a blending function, $\theta$, that yields to the RANS approach when $\theta=1$ and recovers the LES approach for $\theta$ vanishing. Thus, the adopted model can be thought to fall in the class of the blending methods [20,8,2], following the definition in [36]. It allows a natural integration of the VMS concept, [22], which 
allows the eddy-viscosity introduced by the LES closure to be restricted to the smallest resolved scales. This aims at reducing the excessive damping introduced by eddy-viscosity models on the large scales and at giving reasonable predictions with coarser meshes. References $[26,27,25]$ offer a study of the present hybrid approach's impact on subcritical bluff body flow, in particular in combination with the dynamic method of Germano-Lilly [11, 23]. Our motivation in combining RANS with the VMS dynamic formulation is to be able to perform turbulent flow simulations on coarser meshes than with a RANS/LES hybrid model with a comparable accuracy.

Applications involve three test cases. The flow past a cylinder at Reynolds number 140,000 is first studied with the OES-DES approach. The flow past a cylinder at Reynolds number 1M is computed with the hybrid RANS/VMS-Dyn approach. Lastly, the computation of a tandem cylinder is performed by both methods, the DDES model, in which the RANS part has been modified in respect of the turbulence length scale, by using the OES approach, and the hybrid-VMS one.

Table 1: Flow past a cylinder, $\mathrm{Re}=140,000$ in a confined environment of aspect ratio $\mathrm{L} / \mathrm{D}=4.8$ and blockage coefficient $\mathrm{D} / \mathrm{H}=0.208$ in the wind tunnel S4 of IMFT: global parameters

\begin{tabular}{|l|c|r|}
\hline Parameter & simulations & Experiments \\
\hline$C_{D}$ & 1.47 & 1.45 \\
$S_{t}$ & 0.2287 & 0.21 \\
$l_{\text {Rec }}$ & 1.26 & $1.28 / 1.23$ \\
\hline
\end{tabular}

\section{Flow past a circular cylinder at $R e=140,000$ (DDES-OES model)}

Figure 1, left shows an instantaneous view of the $\omega_{z}$ vorticity pattern around the obstacle, obtained by the DDES-OES model. The von Kármán vortices envelop complex smaller-scale structures that fill-up the energy spectrum by a multitude of frequencies due to the turbulent fluctuation. In the shear layers past the separation points, Kelvin-Helmholtz eddies are formed, clearly obtained by the present simulation and turbulence modelling approach. Their temporal and space characteristics will be discussed in the following sections. It is worthwhile noticing that the formation of these smaller-scale organised structures that result from a shearlayer instability, can be damped in cases of using statistical turbulence modelling approaches that produce an excessive turbulence diffusion. Figure 1, right shows the tracking of the shear-layer vortices within a vortex shedding period. The roll-up process of these vortices within the adjacent von Kármán vortex is shown. This merging is a non-linear interaction between the two instabilities and leads to appearance of additional frequency peaks in the energy spectrum as shown Figure 4, by using probe points indicated in Figure 3. The global parameters of the flow are shown in Table 1.

Figure 2, top shows the comparison of the mean drag coefficient, as well as of the mean wall pressure coefficient with experimental studies by [30, 32], the socalled "IMFT circular cylinder" test case, measured by TRPIV2C and TRPIV3C, 

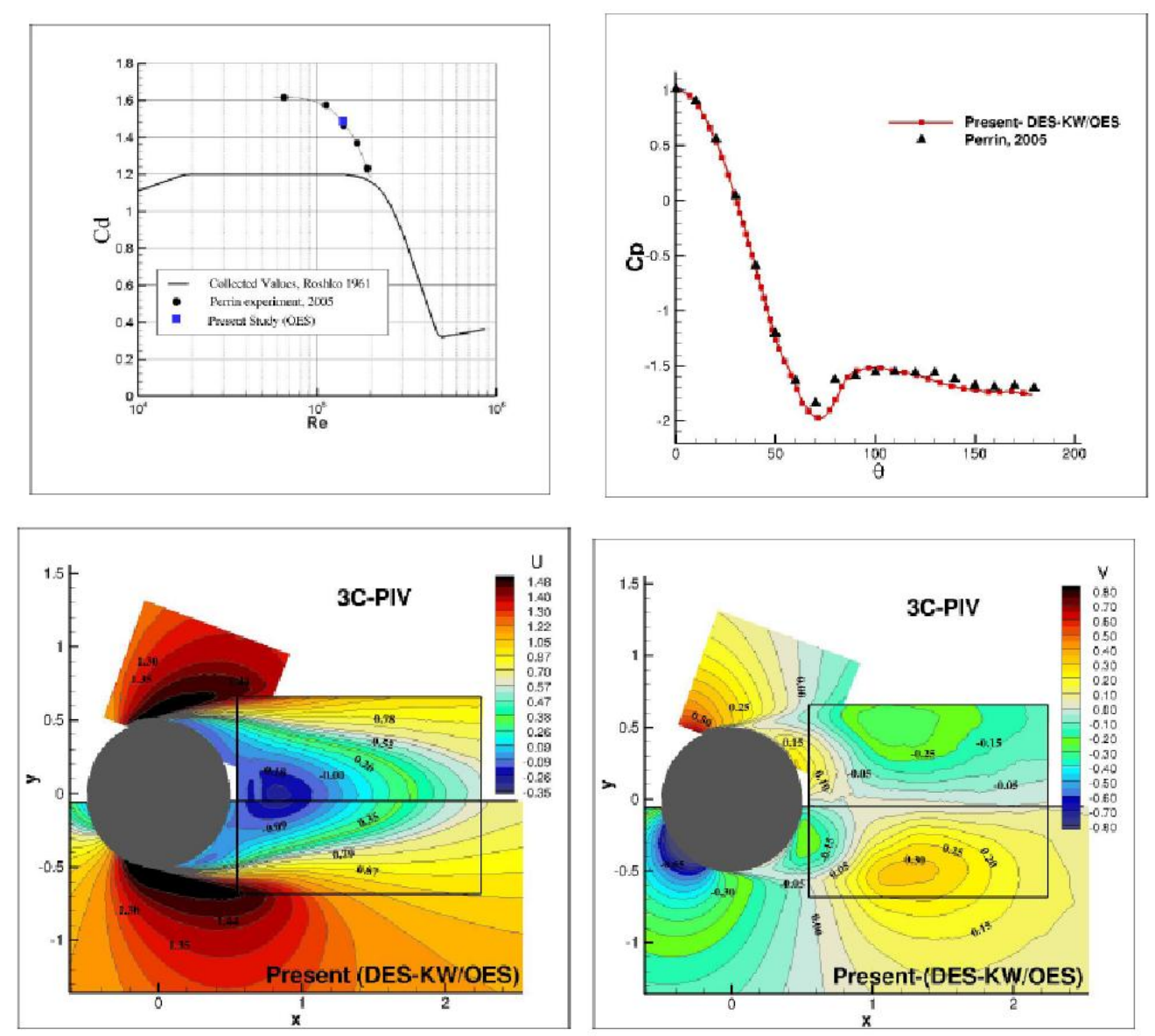

Figure 2: Flow past a cylinder, $R e=140 K$ : top: Mean drag and wall pressure coefficient in comparison with the experiments by Perrin et al (2009). Bottom: Flow past a cylinder, $\mathrm{Re}=140 \mathrm{~K}$ : Comparison of the iso- $\mathrm{U}$ and iso- $\mathrm{V}$ velocity components (averaged) with the PIV experimental data.

Perrin $(2005,2007,2008)$ in the S4 wind tunnel of IMFT (Institut de Mécanique des Fluides de Toulouse, [16]. Figure 2, bottom shows the comparison of the timeaveraged velocity components with the averaged TRPIV experimental data (Perrin, (2005), Perrin et al, (2007)). A good agreement is obtained with the simulations using the DDES-OES modelling. Figure 4 shows the dynamics of the shearlayer instability in association with the von Kármán mode for a point in the shear layer past separation. Morlet's wavelet energy pattern shows concentration of the energy around the Strouhal number of the main vortex shedding, whose value is found 0.226 (also depicted by the FFT spectrum on the right). The presence of the first sub-harmonic also appears, indicating formation of a vortex pairing of the von Kármán eddies, associated with the vortex dislocations phenomenon. The wavelet analysis and the FFT spectrum indicates also formation of an energy pattern around the frequency of 1.37, which corresponds to the shear-layer frequency. Therefore, the ratio between this frequency and the von Kármán mode is found of order 6 . Furthermore, a POD analysis has been carried out in 2D and 3D and compared with POD analysis of the experimental data (Figures 5 and 6). The energy of the 


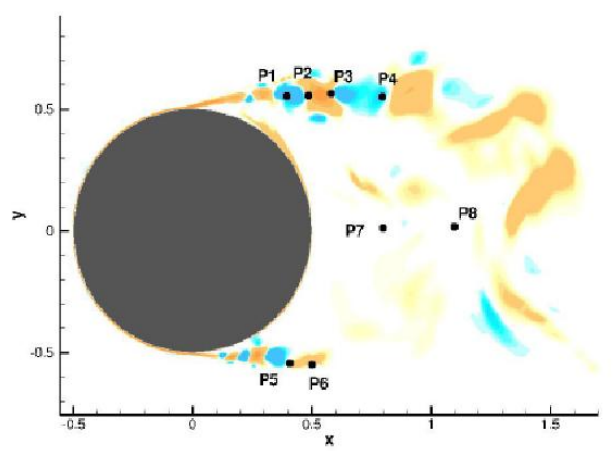

Figure 3: Flow past a cylinder, $R e=140 \mathrm{~K}$ : View of the shear-layer structure and of the Kelvin-Helmholtz vortices past the separation point, as well as of the position of probe points used for the spectral analysis.

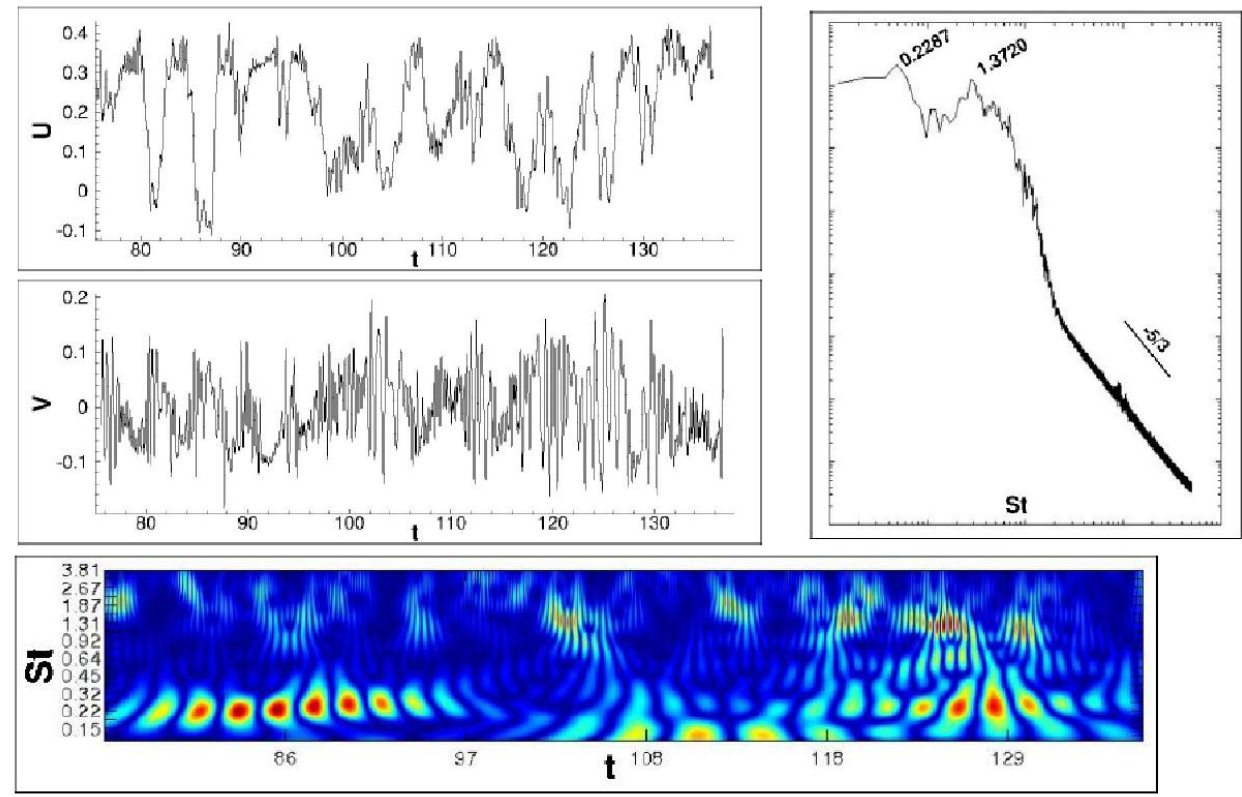

Figure 4: Flow past a cylinder, $R e=140,000$ : Signal processing by using FFT and GrossmanMorlet [14] wavelet analysis for the probe point 3, DDES-OES.

POD modes as a function of the mode's order is presented in Figure 5. A less abrupt slope decrease is remarked for the $3 \mathrm{D}$ case, which has a more rich chaotic statistical content. Furthermore, a slope change in the energy distribution is obtained beyond mode 4 approximately, indicating the progressive influence of random turbulence for the higher mode range. The comparison between the POD modes issued from both approaches is presented on Figure 6. A rather good agreement is shown. It is noticeable that a direct comparison between experimental and simulation POD fields is an original approach in the state of the art concerning the high-Reynolds number circular cylinder flow. 
C. Moussaed et al

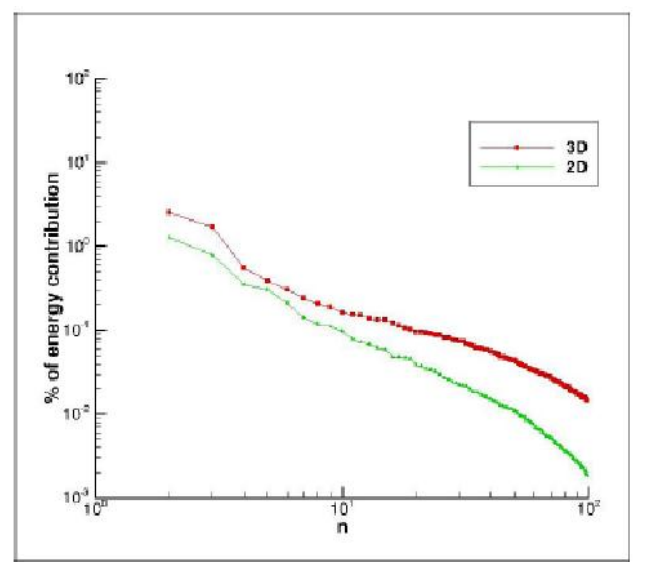

Figure 5: Flow past a cylinder, $\mathrm{Re}=140,000$ : Energy of the POD modes: 2D simulations by the OES approach; 3D simulations by DDES-OES.
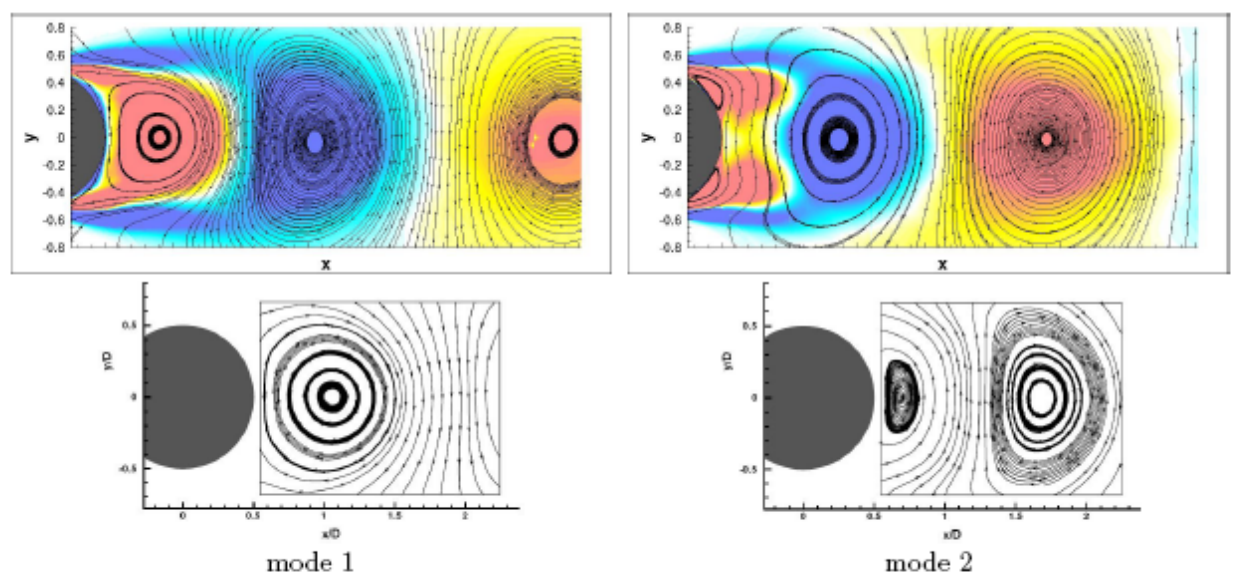

Figure 10. First POD modes; up: present study, streamlines and iso-vorticity contours; bottom: experiment

Figure 6: Flow past a cylinder, $\mathrm{Re}=140,000$ : Comparison of the POD modes between the simulation (DDES-OES) and the experiments (TRPIV).

\section{Flow past a circular cylinder at $R e=1 M$ (RANS/VMS- LES model)}

Only a few numerical investigations have been carried out in the litterature for Reynolds numbers higher than $5 \cdot 10^{5}$. This interval is inside the supercritical regime which appears at Reynolds number higher than $2 \cdot 10^{5}$ and for which the separation becomes turbulent, see [43]. It is generally accepted that periodic vortex shedding is very weak (if any) in the lowest parts of the interval $\left[5 \cdot 10^{5}, 1.5 \cdot 10^{6}\right]$. With increasing Reynolds numbers, the final separation point moves forward, which corresponds to a slight increase of the drag in the supercritical regime. The wall law approach of [33] is used with a distance to the wall $\delta$ set to .002 , which corresponds for 
Table 2: Flow past a cylinder, $\mathrm{Re}=1 \mathrm{M}$ : Bulk flow parameters prediction: $\bar{C}_{d}$ is the mean drag, $C_{l}^{\prime}$ is the root mean square (r.m.s) of the lift coefficient, $\theta$ is the separation angle, $S t$ the vortex shedding frequency made nondimensional by the cylinder diameter and the freestream velocity.

\begin{tabular}{|l|c|c|c|c|c|c|}
\hline & $\begin{array}{c}\text { Mesh } \\
\text { size }\end{array}$ & $\overline{C_{d}}$ & $C_{l}^{\prime}$ & $C p_{\text {base }}$ & St & $\theta$ \\
\hline Present simulations & & & & & & \\
URANS & $1.2 \mathrm{M}$ & 0.24 & 0.06 & 0.25 & 0.46 & 129 \\
LES-VMS & $1.2 \mathrm{M}$ & 0.36 & 0.22 & 0.22 & & \\
Hybrid RANS & $1.2 \mathrm{M}$ & 0.24 & 0.17 & 0.28 & $0.38 / 0.17$ & 131 \\
\hline Simulations & & & & & & \\
RANS of Catalano et al [7] & $2.3 \mathrm{M}$ & 0.39 & & 0.33 & & \\
LES of Catalano et al [7] & $2.3 \mathrm{M}$ & 0.31 & & 0.32 & 0.35 & \\
LES of Ono and Tamura [29] & $4.5 \mathrm{M}$ & 0.27 & 0.13 & & 0.4 & \multirow{2}{*}{0.8} \\
LES of Kim and Mohan [21] & $6.8 \mathrm{M}$ & 0.27 & 0.12 & 0.28 & - & \\
\hline Experiments & & & & & & \\
Shih et al [38] & & 0.24 & & 0.33 & & \\
Schewe [37] & & 0.22 & & & 0.44 & \\
Szechenyi [44] & & 0.22 & & & & \\
Guven et al [15] & & & & & $0.35 / 0.10$ & 130 \\
Goelling [12] & & $0.2-0.4$ & $.1-.15$ & $.2-.34$ & $0.50 / 0.18$ & \\
Zdravkovich [48] & &
\end{tabular}
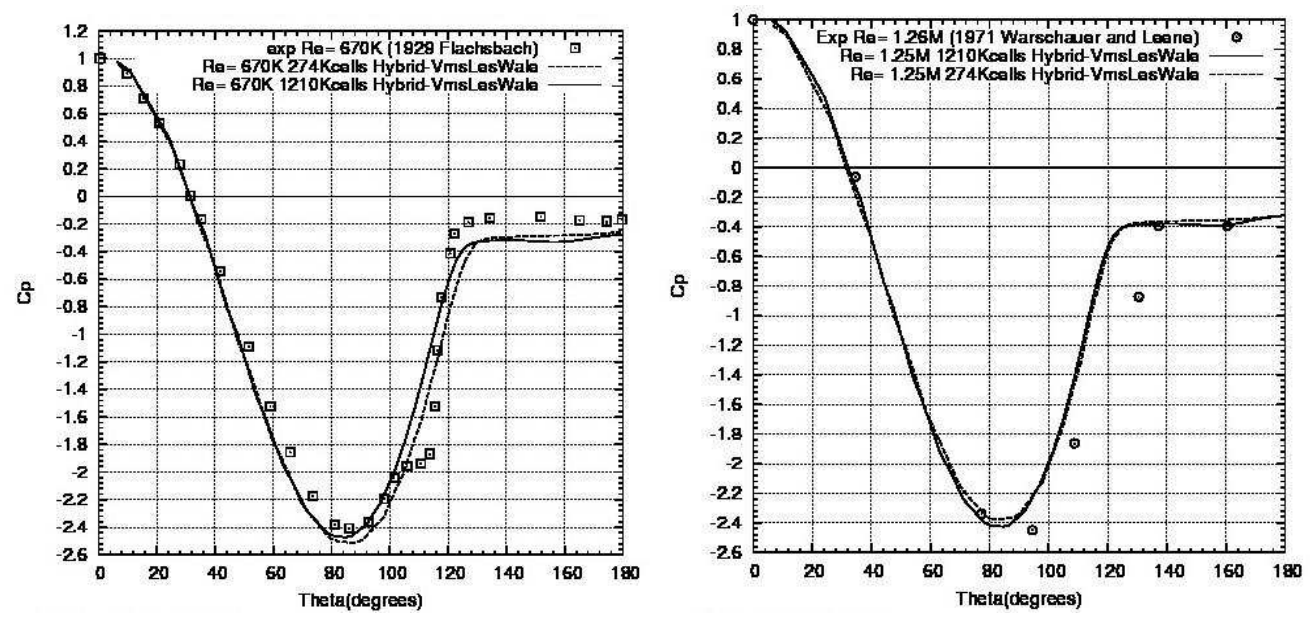

Figure 7: Flow past a cylinder at Reynolds number close to $1 M: C_{p}$ as a function of polar angle. Comparison between experimental data and numerical results obtained on two meshes, of resp. $274 \mathrm{~K}$ cells and $1.21 \mathrm{M}$ cells. Left : Reynolds $670 \mathrm{~K}$, measurements of Flachsbart [35]. Right : Reynolds 1.26M, measurements of Warschauer and Linne [46].

the most part to $y^{+} \simeq 100$. Two rather coarse meshes are used. The first one of $2.74 \cdot 10^{5}$ vertices is a radial mesh with a first layer of vertices of thickness $\delta / 5$, and with a uniform meshing along the azimuthal and spanwise directions. The second one involves $1.2 \cdot 10^{6}$ vertices. It is also a radial mesh, but with a mesh clustering in the azimuthal direction which results in a mesh 4 times finer near the separation and the wake The main outputs are summed up in Table 2. Six 

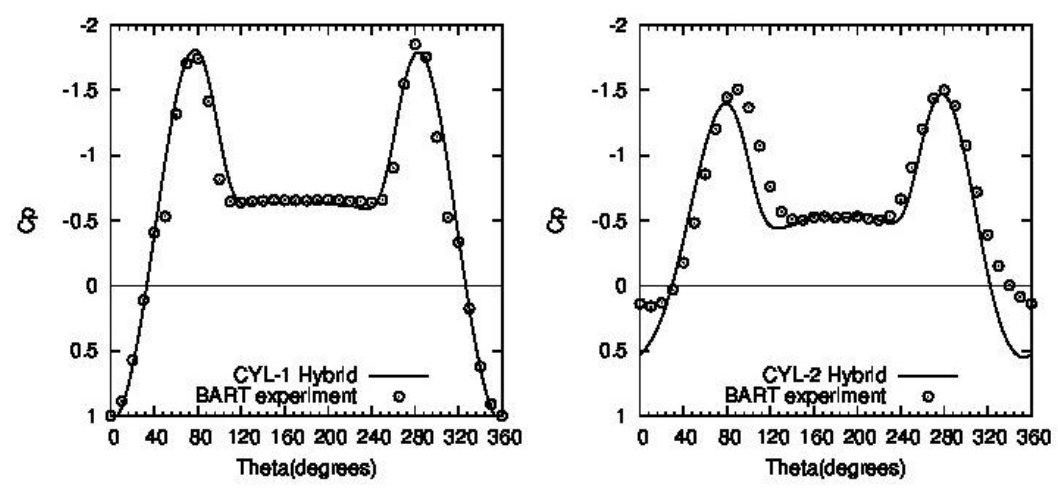

\begin{tabular}{lccc}
\hline & Mesh size & Cyl. 1 & Cyl. 2 \\
& & $\bar{C}_{d}$ & $\bar{C}_{d}$ \\
\hline \hline RANS-VMS dyn. & $2.59 \mathrm{M}$ & 0.64 & 0.38 \\
\hline Simulations & & & \\
Workshop [24] & $2-133 \mathrm{M}$ & $0.33-0.80$ & $0.29-0.52$ \\
DES Aybay [24] & $6.7 \mathrm{M}$ & 0.64 & 0.44 \\
HRLES [45] & $8.7 \mathrm{M}$ & 0.64 & 0.45 \\
\hline Experiments & & & \\
From $C_{p}$ of [28] & & 0.64 & 0.31 \\
\hline
\end{tabular}

Figure 8: Tandem cylinder at $\mathrm{Re}=166 \mathrm{~K}$ with RANS-VMS-Dynamic: top: pressure distribution on both cylinders. Bottom: bulk quantities.

publications giving measurements,including the synthetic book of Zdravkovich [48] were found. The results are compared with three computations with LES models, by Kim and Mohan [21] and Catalano et al [7], Ono and Tamura [29], and a RANS result in [7]. The present RANS calculation provide results which differ importantly from the RANS results produced in [7] with a similar model. Our RANS prediction of drag is rather good, but the Strouhal number predicted is slightly higher than in the experiments experiments, and the $C_{l}^{\prime}$ is 2-3 times lower. The LES simulations of the above references are carried out on grids having $2.3 \cdot 10^{6}$ [7], $4.5 \cdot 10^{6}$ nodes [29], and $6.8 \cdot 10^{6}$ nodes [21]. The LES computations need generally a rather high number of nodes. With less than $4 \mathrm{M}$ nodes, the mean drag is over-estimated. The fine mesh computations provide a rather coherent prediction of the $C_{l}^{\prime}$, at $0.12-0.13$. With the present quite coarse mesh the VMS-LES calculation gives values of drag and $C_{l}^{\prime}$ too high. The hybrid RANS/VMS-LES simulations give rather accurate predictions in terms of mean drag, base pressure and Strouhal number already on the coarser grid. For comparison purpose with available $C_{p}$ measurements $[35,46]$, the present flow has been recomputed through the hybrid RANS/VMS-LES approach at Reynolds numbers $6.7 \cdot 10^{5}$ and $1.25 \cdot 10^{6}$, see Figure 7 . For the case at $R e=6.7 \cdot 10^{5}$ in Figure $7 \mathrm{a}$, there is a slight under-estimation of the base pressure. Moreover, in the experimental curve there is a kink at $\theta=110^{\circ}$, which indicates the presence of a small secondary recirculation bubble. This separation bubble is not captured in the present calculations. It is not either captured in [7], but a separation is found in [29]. At $R e=1.25 \cdot 10^{6}$, the base pressure is quite well predicted, while it seems that the separation occurs more upstream than in the experiments. 


\section{RANS-VMS simulation of tandem cylinder at $\mathrm{Re}=166,000$}

The tandem cylinder configuration was used as a benchmark case in the AIAA workshop on Benchmark problems for Airframe Noise Computations (BANC I/II) [34,35] and in the 7th EU framework project Advanced Turbulence simulation for Aerodynamic Application Challenges (ATAAC)[36,37]. The Reynolds number is 166, 000 and pitch distance (between the cylinders centers) of 3.7 has been considered. The experimental results by statistical (not time-resolved PIV) were obtained by Jenkins et al [19] in the NASA-Langley research center. In Figure 8 the results of a computation with a mesh of $2.3 \mathrm{M}$ vertices using the RANS-VMS model are presented.

\section{DDES-OES simulation of tandem cylinder $\mathrm{Re}=166,000$}

This second study aims at detecting the modification of the shear-layer mode past the second cylinder, responsible for acoustic noise in the phases of landing gear configuration. In the context of the European program ATAAC, a mesh of order $16 \mathrm{M}$ points has been employed, provided by M. Strelets and M. Shur of St Petersbourg (ATAAC partners). The computations have been carried out by the DDES-OES modelling (Bourguet et al 2008), (Braza et al, 2006). Figure 10 (left) presents the comparison of the simulated averaged velocity field with the corresponding PIV measured velocity field. A good agreement is obtained. Figure 10 (right) presents the comparison of the pressure coefficient around the downstream cylinder. A reasonably good agreement is obtained. In the median region between the cylinders, the agreement would be improved by using an even finer mesh. Figure 9, left and 10 show the complex turbulence structure around the obstacles simultaneously governed by coherent eddies and chaotic turbulence motion. The coherent flow pattern corresponds to the von Kármán mode and to Kelvin-Helmholtz vortices, both associated with acoustic noise of a landing gear. The present modelling has been able to predict these modes, as well as the secondary instability related with spanwise-periodic vortex bursts (Figure 11). The shear-layer vortices and the overall development of the related instability pattern past the upstream and downstream cylinder are clearly illustrated (Figure 10). The comparison of the averaged velocity fields is compared
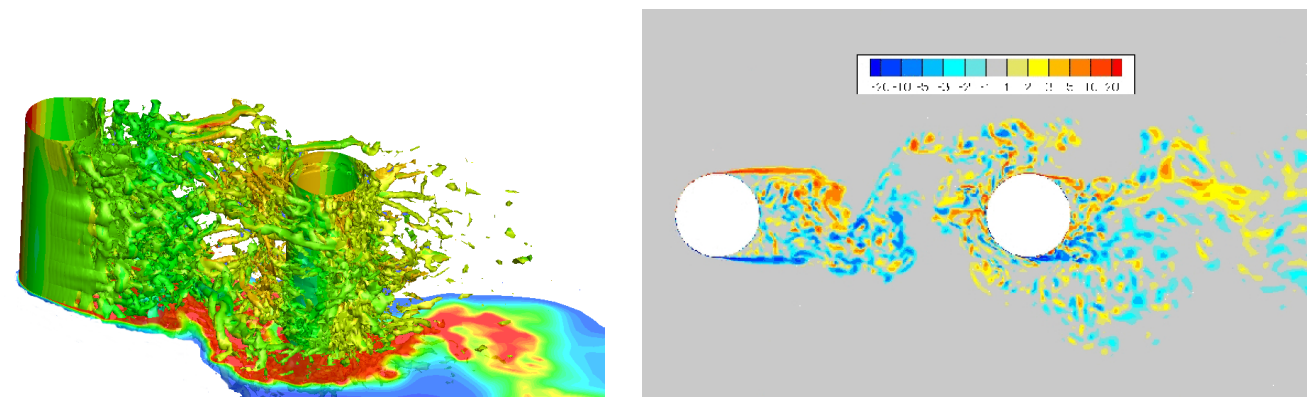

Figure 9: Tandem cylinder ar $\operatorname{Re}=166,000$ : Left: Iso-vorticity contours coloured by pressure around a tandem cylinder configuration, by means of the DDES-OES model. Right: Tandem cylinder ar Re=166K: Iso-vorticity contours - median plane. 

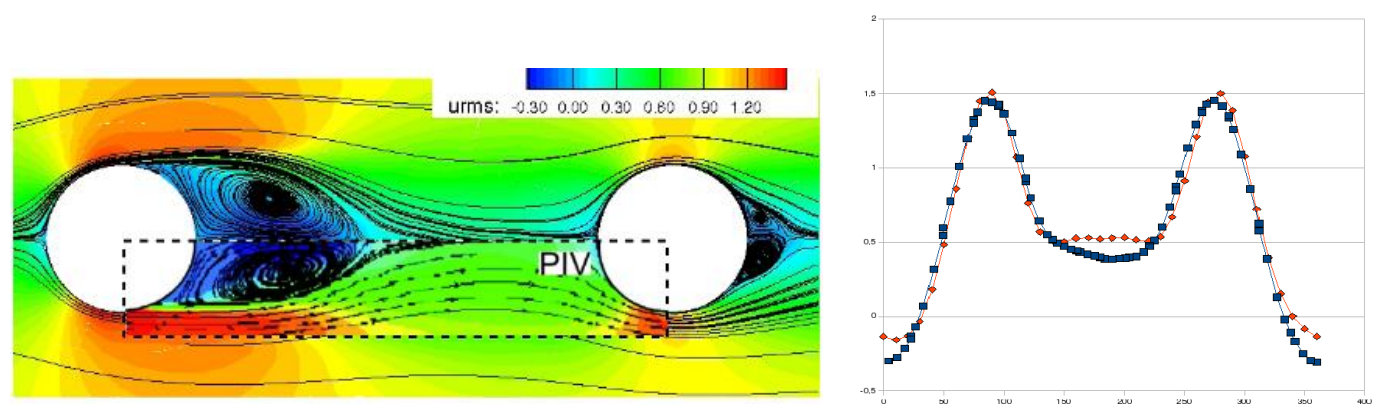

Figure 10: Tandem cylinder ar $\mathrm{Re}=166,000$ : Left: comparison of the averaged streamlines and velocity fields with the experiments of [19]. Right: Averaged pressure coefficient around the second cylinder.

with the experimental results by Jenkins et al (2005) - NASA Langley research center and they are found in good agreement. Figure 9, right presents a median plane of the complex vortex structure where the von Kármán and Kelvin-Helmholtz eddies can be distinguished. Figure 11 (left) presents an almost horizonal view from above of the flow around the tandem cylinders configuration, illustrating the complexity of the smaller-scale turbulence structures resolved by the present DDES-OES method, as well as of the spanwise vortex structure, characterised by secondary instability bursts. Figure 11 right presents the comparison of the energy spectrum with the afore mentioned experiments. A good prediction of the von Kármán mode (first predominent frequency peak) and of the Kelvin-Helmholtz shear-layer mode (second predominent peak) regarding the experimental spectrum is shown.
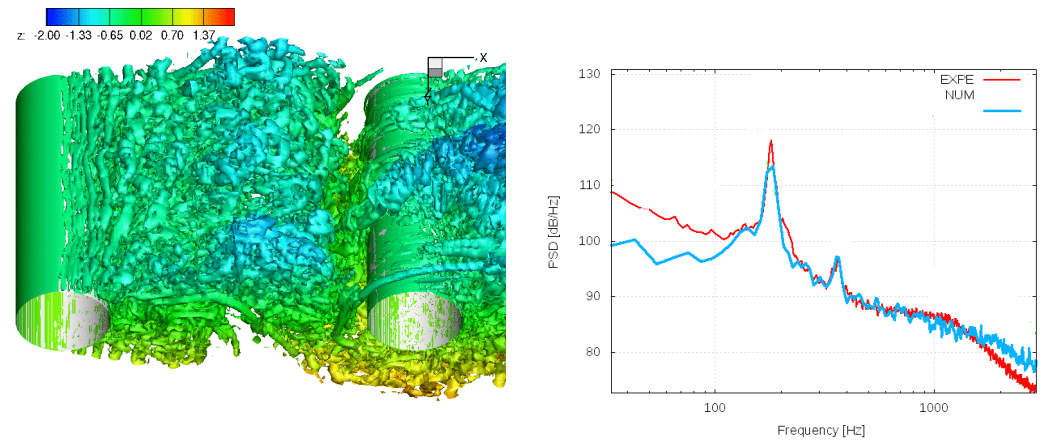

Figure 11: Tandem cylinder ar $R e=166,000$ : Left: Turbulence structure between the two cylinders and spanwise negative vorticity filaments (blue) around the downstream cylinder, DDES-OES. Right: pressure spectrum at point located at $45^{\circ}$ clockwise on the downstream cylinder. Comparison of the Kelvin-Helmholtz frequency peaks, responsible for the landing gear acoustic noise, with the experiments of the NASA-Ames research center. 


\section{Conclusions}

The combination of tractable LES and RANS models may result in terms of an excessively damping large scales, either in region of LES in which the back-scatter needs to be preserved, or in non-equilibrium turbulence regions in which URANS modelling is insufficient, because of the use of downscale turbulence cascade assumptions.

A strategy for blending RANS and VMS-LES has been applied to the simulation of the flow around bluff bodies. In this hybrid model, a blending function based on the values of a blending parameter is introduced, in order to automatically switch from RANS to LES. This allows to replace the LES component by a novel dynamic VMS model.

This hybrid method has been applied to the simulation of the flow around a circular cylinder at Reynolds number 1M. It was shown that bulk quantities can be reasonably predicted even with the use of a quite coarse mesh in the case of the RANS/VMS-LES hybridization, contrarily to the RANS model because of a too poor flow-physics modeling and contrarily to the VMS-LES approach which needs finer grids.

The flow around a tandem cylinder at Reynolds number 166, 000 has been simulated by the same hybrid model, RANS/VMS-LES, by using in this case also, a rather coarse mesh and allows to obtain rather good pressures and bulk coefficients.

In order to emphasize the capturing of near-wall non-equilibrium turbulence and to accurately predict the instability patterns and predominent frequencies governing the present flow, a DDES model where the RANS part has been modified in respect of the turbulence length scale, by using the OES approach, has been applied to the computation of the flow past a cylinder entering the critical regime and tandem cylinder configurations. The present study has shown that the turbulence modelling approach DDES-OES is able to capture the main instabilities in the complex turbulence structure around a single and tandem cylinders configuration in the high Reynolds number range in good agreement with the physical experiments. Moreover, the frequencies of the main instability modes as well as the global parameters are predicted quite well by the present method.

\section{References}

[1] Progress in Hybrid RANS-LES modelling. In Notes on Numerical Fluid Mechanics and Multidisciplinary Design, 130. Springer, 2015.

[2] R.A. Baurle, C.-J. Tam, J.R. Edwards, and H.A. Hassan. Hybrid simulation approach for cavity flows: Blending, algorithm, and boundary treatment issues. AIAA J., 41(8):14631480, 2003.

[3] P. W. Bearman. Investigation of the flow behind a two-dimensional model with a blunt trailing edge and fitted with splitter plates. Journal of Fluid Mechanics, 21:241-255, 1965.

[4] R. Bourguet, M. Braza, G. Harran, and R. El Akoury. Anisotropic organised eddy simulation for the prediction of non-equilibrium turbulent flows around bodie. J. Fluids and Structures, $24(8): 1240-1251,2008$.

[5] M. Braza, R. Perrin, and Y. Hoarau. Turbulence properties in the cylinder wake at high Reynolds number. J. Fluids and Structures, 22(6):757-771, 2006.

[6] B. Cantwell and D. Coles. An experimental study of entrainment and transport in the turbulent near wake of a circular cylinder. Journal of Fluid Mechanics, 136:321-374, 1983. 
[7] P. Catalano, Meng Wang, G. Iaccarino, and P. Moin. Numerical simulation of the flow around a circular cylinder at high Reynolds numbers. Int. J. Heat Fluid Flow, 24:463-469, 2003.

[8] C.-C. Fan, X. Xiao, J.R. Edwards, and H.A. Hassan. Hybrid large-eddy/Reynolds-averaged navier-stokes simulations of shock-separated flows. J. Spacecraft Rockets, 41(6):897-906, 2004.

[9] J. Froehlich and D. von Terzi. Hybrid LES/RANS methods for the simulation of turbulent flows. Prog. Aerosp. Sci., 44(5):349-377, 2008.

[10] T. B. Gatski and C. G Speziale. On explicit algebraic stress models for complex turbulen flows. J. Fluid Mech., 254:59-78, 1993.

[11] M. Germano, U. Piomelli, P. Moin, and W. Cabot. A dynamic subgrid-scale eddy viscosity model. Phys. Fluids A, 3(7):1760-1765, 1991.

[12] B. Goelling. Experimental investigations of separating boundary-layer flow from circular cylinder at Reynolds numbers from $10^{5}$ up to $10^{7}$; three-dimensional vortex flow of a circular cylinder. In G.E.A. Meier and K.R. Sreenivasan, editors, Proceedings of IUTAM Symposium on One Hundred Years of Boundary Layer Research, pages 455-462, The Netherlands, 2006. Springer.

[13] F. Grossi, M. Braza, and Y. Hoarau. Prediction of transonic buffet by delayed detached-eddy simulation. AIAA J., 52(10):2300-2312, 2014.

[14] A. Grossmann and J. Morlet. Decomposition of Hardy functions into square integrable wavelets of constant shape. SIAM J., 15:723-736, 1984.

[15] O. Guven, C. Farell, and V.C. Patel. Surface-roughness effects on the mean flow past circular cylinders. J. Fluid Mech., 98(4):673-701, 1980.

[16] W. Haase, M. Braza, and A. Revell. DESider - a European effort on hybrid RANS-LES modelling. Notes on Numerical Fluid Mechanics and Multidisciplinary Design, 103, 2009.

[17] T.J.R. Hughes, L. Mazzei, and K.E. Jansen. Large eddy simulation and the variational multiscale method. Comput. Vis. Sci., 3:47-59, 2000.

[18] J. Chiva R. Borrell I. Rodriguez, O. Lehmkuhl and A. Oliva. On the flow past a circular cylinder from critical to super-critical reynolds numbers: wake topology and vortex shedding. In Proceeedings of ETMM10, Marbella, Spain, September 2013.

[19] L.N. Jenkins, M. R. Khorrami, M. M. Choudhari, and C. B. McGinley. Characterization of unsteady flow structures around tandem cylinders for component interaction studies in airframe noise, AIAA 2005-2812, 2005.

[20] S. Kawai and K. Fujii. Analysis and prediction of thin-airfoil stall phenomena with hybrid turbulence methodology. AIAA J., 43(5):953-961, 2005.

[21] S.-E. Kim and L.S. Mohan. Prediction of unsteady loading on a circular cylinder in high Reynolds number flows using large eddy simulation. In Proceedings of OMAE 2005: 24th International Conference on Offshore Mechanics and Artic Engineering, Halkidiki, Greece, june 12-16 2005. OMAE 2005-67044.

[22] B. Koobus and C. Farhat. A variational multiscale method for the large eddy simulation of compressible turbulent flows on unstructured meshes-application to vortex shedding. Comput. Methods Appl. Mech. Eng., 193:1367-1383, 2004.

[23] D.K. Lilly. A proposed modification of the Germano subgrid-scale closure method. Phys. Fluids, A4:633, 1992.

[24] D.P. Lockard. Summary of the tandem cylinder solutions from the benchmark problems for airframe noise computations-I. In Proceedings of 49th AIAA Aerospace Sciences Meeting, AIAA-2011-353, Orlando, Florida, Jan 2011.

[25] C. Moussaed, M.V. Salvetti, S. Wornom, B. Koobus, and A. Dervieux. Simulation of the flow past a circular cylinder in the supercritical regime by blending RANS and variationalmultiscale LES models. Journal of Fluids and Structures, 47:114-123, 2014. 
[26] C. Moussaed, S. Wornom, B. Koobus, M.-V. Salvetti, and A. Dervieux. Dynamic variational multiscale LES of bluff body flows on unstructured grids. In ICCFD 2013, International Conference on Computational Fluid Dynamics, Berlin, Germany, May 22-23 2013.

[27] C. Moussaed, S. Wornom, M.V. Salvetti, B. Koobus, and A. Dervieux. Impact of dynamic subgrid-scale modeling in variational multiscale large-eddy simulation of bluff body flows. Acta Mechanica, 12:3309-3323, 2014.

[28] D. Neuhart, L. Jenkins, M. Choudhari, and M. Khorrami. Measurements of the flowfield interaction between tandem cylinders. AIAA Paper, AIAA-2009-3275, 2009.

[29] Y. Ono and T. Tamura. LES of flows around a circular cylinder in the critical Reynolds number region. In Proceedings of BBAA VI International Colloquium on Bluff Bodies Aerodynamics and Applications, Milano, Italy, July 20-24 2008.

[30] R. Perrin. Analyse physique et modélisation d'écoulements incompressibles instationnaires turbulents autour d'un cylindre circulaire à grand nombre de Reynolds, $\mathrm{PhD}$ thesis, Institut National Polytechnique de Toulouse, 5 july 2005.

[31] R. Perrin, M. Braza, E. Cid, S. Cazin, F. Thiele, and J. Borée. Time resolved stereoscopic PIV measurements in the near wake of a circular cylinder at high Reynolds number. In Proceedings of 14th Int Symp on Applications of Laser Techniques to Fluid Mechanics, Lisbon, Portugal, 7-10 July 2008.

[32] R. Perrin, E. Cid, Cazin S., Sevrain A., Braza M., F. Moradei, and G. Harran. Phaseaveraged measurements of the turbulence properties in the near wake of a circular cylinder at high Reynolds number by 2C-PIV and 3C-PIV. Experiments in Fluids, 42(1):93-109, 2007.

[33] H. Reichardt. Vollstaendige Darstellung der turbulenten Geschwindigkeitsverteilung in glatten Leitungen. Zeitschrift fuer Angewandte Mathematik und Mechanik, 31:208, 1951.

[34] S. Rolfo and A. Revell. Effect of span-wise resolution for LES of flow over a rotating cylinder at high Reynolds number. In ERCOFTAC symposium Unsteady separation in fluid-structure interaction, Mykonos, 2013.

[35] A. Roshko. Experiments on the flow past a circular cylinder at very high Reynolds number. J. Fluid. Mech., 10:345-356, 1961.

[36] P. Sagaut, S. Deck, and M. Terracol. Multiscale and multiresolution approaches in turbulence. Imperial College Press, London, 2006.

[37] J.W. Schewe. On the forces acting on a circular cylinder in cross flow from subcritical up to transcritical Reynolds numbers. J. Fluid Mech., 133:265-285, 1983.

[38] W.C.L. Shih, C. Wang, D. Coles, and A. Roshko. Experiments on flow past rough circular cylinders at large Reynolds numbers. J. Wind Eng. Indust. Aerodyn., 49:351-368, 1993.

[39] V. Shinde, T. Marcel, Y. Hoarau, T. Deloze, G. Harran, F. Baj, J.Cardolaccia, J.P. Magnaud, E. Longatte, and M. Braza. Numerical simulation of the fluid structure interaction in a tube array under cross flow at moderate and high Reynolds number. J. of Fluids and Structures, 47:99-113, 2014.

[40] P. Spalart, S. Deck, M. Shur, K. Squires, M. Strelets, and A. Travin. A new version of detached-eddy simulation, resistant to ambiguous grid densities. Theory and Computational Fluid Dynamics, 20:181-195, 2006.

[41] P.R. Spalart, W.H. Jou, M. Strelets, and S. Allmaras. Advances in DNS/LES, chapter Comments on the feasibility of LES for wings and on a hybrid RANS/LES approach. Columbus $(\mathrm{OH}), 1997$.

[42] P.R. Spalart and M. Shur. On the sensitization of simple turbulence models to rotation and curvature. Aerospace Sci. Technol., 1(5):297, 1997.

[43] B.M. Sumer and J. Fredsoe. Hydrodynamics around cylindrical structures. World Scientific, 2006. 
C. Moussaed et al

[44] E. Szechenyi. Supercritical Reynolds number simulation for two-dimensional flow over circular cylinders. J. Fluid Mech., 70:529-542, 1975.

[45] V.N. Vatsa and D.P. Lockard. Assessment of hybrid RANS/LES turbulence models for areoacoustics applications. AIAA Paper, AIAA-2010-4001, 2010.

[46] K. A. Warschauer and J. A. Leene. Experiments on mean and fluctuating pressures of circular cylinders at cross flow at very high Reynolds numbers. Proc. Int. Conf. on Wind Effects on Buildings and Structures, Saikon, Tokyo, 305-315 (see also Zdravkovich 1997), 1971.

[47] C. Wieselsberger. Neuere Feststellungen iiber die Gesetze des Fliissigkeits- und Luftwiderstands. Physikalische Zeitschrift, 2:321-328, 1921.

[48] M.M. Zdravkovich. Flow around circular cylinders. Vol 1: Fundamentals. Oxford Science Publications. Oxford University Press, 1997. 